\section{THE AMERICANJOURNALOF}

\section{PSYCHIATRY}

\section{Contents}

\section{January 2015, Volume 172}

\section{Editorials}

1 Insurance, stigma, and women's mental health Gail Erlick Robinson

3 Use of electroconvulsive therapy in bipolar depression Mauricio Tohen

6 The prevention of detention David A. Brent and Rolf Loeber

9 Can behavioral health organizations change health behaviors? The STRIDE study and lifestyle interventions for obesity in serious mental illness Stephen J. Barteis

\section{Clinical case conference}

12 Hypercalcemia and 'primary' hyperparathyroidism during lithium therapy

Howard I. Shapiro and Karina A. Davis

\section{Images in psychiatry}

16 Pigeon Cave: a legendary place of suicide in Lebanon Sami Richa and Nathalie Richa

\section{Reviews and overviews}

17 Sensory processing dysfunction in the personal experience and neuronal machinery of schizophrenia Daniel C. Javitt and Robert Freedman

\section{New research - articles}

32 A randomized trial of collaborative depression care in obstetrics and gynecology clinics: socioeconomic disadvantage and treatment response Wayne Katon, Joan Russo, Susan D. Reed, Carmen A. Croicu, Evette Ludman, Anna LaRocco, Jennifer L. Melville

41 Treatment-resistant bipolar depression: a randomized controlled trial of electroconvulsive therapy versus algorithm-based pharmacological treatment Helle K. Schoeven, Ute Kessler, Ole A. Andreassen, Bjoern H. Auestad, Per Bergsholm, Ulrik F. Mait, Gunnar Morken, Ketit J. Oedegaard, Arne vaaler
Electroconvulsive therapy augmentation in clozapine-resistant schizophrenia: a prospective, randomized study

Georgios Petrides, Chitra Malur, Raphael J. Braga, Samuel H. Bailine, Nina R. Schooler, Anil K. Malhotra, John M. Kane, Sohag Sanghani, Terry E. Goldberg, Majnu John, Alan Mendelowitz

Impact of early intervention on psychopathology crime, and well-being at age 25 Kenneth A. Dodge, Karen L. Bierman, John D. Coie, Mark T. Greenberg, John E. Lochman, Robert J. McMahon, Ellen E. Pinderhughes, for the Conduct Problems Prevention Research Group

71 The STRIDE weight loss and lifestyle intervention for individuals taking antipsychotic medications: a randomized trial

Carla A. Green, Bobbi Jo $\mathrm{H}$. Yarborough, Michael C. Leo, Mican T. Yarborough, Scott P. Stumbo, Shannon L. Janoff Nancy A. Perrin, Greg A. Nichols, Victor J. Stevens

82 Cross-disorder genome-wide analyses suggest a complex genetic relationship between Tourette's syndrome and $O C D$

Dongme Yu, Carol A. Mathews, Jeremiah M. Scharf, Benjamin M. Neale, Lea K. Davis, Eric R. Gamazon, Eske M. Derks, Patrick Evans, Christopher K. Edlund, Jacquelyn Crane, Jesen A. Fagerness, Lisa Osiecki, Patience Gallagher, Gloria Gerber, Stephen Haddad, Cornelia IIImann, Lauren M. McGrath, Catherine Mayerfeld, Sampath Arepalli, Cristina Barlassina, Cathy L. Barr, Laura Beltodi, Fortu Benarroch, Gabriel Bedoya Berrió, O. Joseph Bienvenu, Donald W. Black, Michael $\mathrm{H}$. Bloch, Helena Brentani, Ruth D. Bruun, Cathy L. Budman, Beatriz Camarena, Desmond D. Campbell, Carolina Cappi, Julio C. Cardona Silgado, Maria C. Cavallini, Denise A. Chavira, Sylvain Chouinard, Edwin $\mathrm{H}$. Cook, M. R. Cookson, Vladimir Coric, Bernadette Cutlen, Daniele Cusi, Richard Delorme, Damiaan Denys, Yves Dion, Valsama Eapen, Karin Egberts, Peter Falkai, Thomas Fernandez, Eduardo Fournier, Helena Garrido, Danief Geller, Donald L. Gilbert, Simon L. Girard, Hans J. Grabe, Marco A. Grados, Benjamin D. Greenberg, Varda Gross-Tsur, Edna Grünblatt, John Hardy, Gary A. Heiman, Sian M.J. Hemmings, Luis D. Herrera, Dianne M. Hezel, Pieter J. Hoekstra, Joseph Jankovic, James L. Kennedy, Robert A. King, Anuar I. Konkashbaev, Barbara Kremeyer, Roger Kurlan, Nuria Lanzagorta, Marion Leboyer, James F. Leckman, Leonhard Lennertz, Chunyu Liu, Christine Lochner, Thomas L. Lowe, Sara Lupoli, Fabio Macciardi Wolfgang Maier, Paolo Manunta, Maurizio Marconi, James T. Mccracken, Sandra C. Mesa Restrepo, Rainald Moessner Priya Moorjani, Jubel Morgan, Heike Mutler, Dennis L. Murphy, Allan L. Naarden, Erika Nurmi, William Cornejo Ochoa, Roel A. Ophoff, Andrew J. Pakstis, Michele T. Pato, Carlos N. Pato, John Piacentini, Christopher Pittenger, Yehuda Pollak, Scott L. Rauch, Tobias Renner, victor f. Reus, Margaret A. Richter Mark A. Riddle, Mary M. Robertson, Roxana Romero, Maria C. Rosário, David Rosenberg, Stephan Ruhrmann, Chiara Sabatti, Erika Salvi, Aline S. Sampaio, Jack Samuels, Pauł Sandor, Susan K. Service, Brooke Sheppard, Harvey S. Singer Jan H. Smit, Dan J. Stein, Eric Strengman, Jay A. Tischfield, Maurizio Turiel, Ana V. Valencia Duarte, Homero Valtada, Jeremy Veenstra-VanderWeele, Susanne Walitza, Ying Wang, Mike Weale, Robert Weiss, Jens R. Wendland, Herman G. M. Westenberg, Yin Yao Shugart, Ana G. Hounie, Euripedes C. Miguel, Humberto Nicolint, Michael Wagner. Andres Ruiz-Linares, Danielle C. Cath, William McMahon, Danielle Posthuma, Ben A. Oostra, Gerald Nestadt, Guy A. Rouleau, Shaun Purcell, Michael A. Jenike, Peter Heutink, Gregory L. Hanna, David V. Conti, Paul D. Arnold, Neison B. Freimer,

S. Evelyn Stewart, James A. Knowles, Nancy I. Cox, David L. Pauls 